\title{
Infección por Fasciola hepatica en escolares del distrito de Condebamba, Cajamarca
}

\author{
Fasciola hepatica infection in schoolchildren of Condebamba district, Cajamarca \\ Claudia Rodríguez-Ulloa ${ }^{1,4}$, Marco Rivera-Jacinto ${ }^{1}$, Silvia Chilón Y. ${ }^{1}$, Pedro Ortiz O. ${ }^{2}$, \\ Juana Del Valle-Mendoza ${ }^{3}$
}

\section{Resumen}

La investigación tuvo como finalidad determinar la prevalencia de Fasciola hepatica y sus factores de riesgo en escolares de instituciones educativas del nivel primario de tres caseríos del distrito de Condebamba, Cajamarca, Perú. Se aplicó un cuestionario a los escolares y a sus padres o apoderados para recolectar información relacionada con los factores de riesgo en los escolares. Se evaluaron 253 muestras fecales de escolares entre 6 y 12 años, mediante la técnica de sedimentación rápida modificada por Lumbreras para diagnóstico de infección por $F$. hepatica. La prevalencia de $F$. hepatica en Condebamba fue de $5.1 \%$. No hubo diferencias significativas en relación con el género y grupo de edad. Los factores de riesgo asociados con la infección fueron la crianza de vacas y defecar al aire libre. Las prevalencias fueron moderadas en las localidades de Cauday y Ogosgón y nula en La Merced.

Palabras clave: Fasciola hepatica; fascioliasis humana; prevalencia; factores de riesgo; epidemiología

\section{Abstract}

The purpose of this research was to determine the prevalence of Fasciola hepatica and its risk factors in schoolchildren of educational institutions of the primary level of three villages in the district of Condebamba, Cajamarca, Peru. Aquestionnaire was applied

${ }^{1}$ Departamento de Ciencias Biológicas, Facultad de Ciencias de la Salud, Universidad Nacional de Cajamarca, Perú

${ }^{2}$ Departamento de Ciencias Veterinarias, Facultad de Ciencias Veterinarias, Universidad Nacional de Cajamarca, Perú

${ }^{3}$ Centro de Investigación de la Facultad de Ciencias de la Salud, Universidad Peruana de Ciencias Aplicadas, Lima, Perú

${ }^{4}$ E-mail: claudiarodriguez@unc.edu.pe

Recibido: 9 de febrero de 2018

Aceptado para publicación: 24 de julio de 2018 
to schoolchildren and their parents or guardians to collect information related to risk factors in schoolchildren. It was evaluated 253 faecal samples of schoolchildren between 6 and 12 years old, using the rapid sedimentation technique modified by Lumbreras to diagnose $F$. hepatica infection. The prevalence of $F$. hepatica in Condebamba was $5.1 \%$. There were no significant differences in relation to gender or age group. The risk factors associated with the infection were raising cows and defecating outdoors. The prevalence was moderate in Cauday and Ogosgón villages and null in La Merced.

Key words: Fasciola hepatica; human fascioliasis; prevalence; risk factors; epidemiology

\section{INTRODUCCIÓN}

Fasciola hepatica, uno de los agentes causales de la fascioliasis, afecta las actividades ganaderas a nivel mundial causando millonarias pérdidas económicas, además de su impacto sobre la salud humana (Cwiklinski et al., 2016). Esta importante zoonosis es clasificada por la Organización Mundial de la Salud (OMS) como una de las enfermedades más desatendidas, y debido a su amplia distribución como trematodiasis de transmisión alimentaria, afecta al humano en todos los continentes (excepto la Antártida) (MasComa, 2005).

En el Perú, la fascioliasis es un problema veterinario de importancia por las altas tasas de infección del ganado en la mayoría de las regiones del país y un problema de salud pública por la alta prevalencia de la infección humana (Espinoza et al., 2010). La transmisión humana ocurre principalmente en las poblaciones rurales dedicadas a la agricultura a lo largo de los valles y pendientes andinas, hasta los $4500 \mathrm{msnm}$ (Cabada et al., 2014). En los últimos años, en Cajamarca, una de las principales zonas endémicas de fascioliasis en ganado, se ha observado un constante incremento en el número de casos humanos (Marcos y Terashima, 2007; González et al., 2011), siendo los niños en edad escolar uno de los grupos mayormente afectados; no obstante, debido a la baja sensibilidad de las pruebas coproparasitológicas y a que la parasitosis no es de notificación obligatoria ni tiene sintomatología patognomónica, es posible que muchos de los casos no sean detectados y la incidencia sea mucho mayor (Mas-Coma, 2005; Marcos y Terashima 2007).

Entre las dificultades que existen para el control de la fascioliasis humana está la compleja interacción de los múltiples factores que determinan su transmisión, tales como los factores geográficos y ecológicos del área, factores socio-culturales y económicos de la población, y las características biológicas y ecológicas del hospedero intermediario, entre otros (Mas-Coma et al., 1999). En los últimos años, investigaciones epidemiológicas en zonas endémicas a nivel nacional (Marcos et al., 2004, 2006) e internacional (Curtale et al., 2003; Fentie et al., 2013; ZumaqueroRios et al., 2013), han identificado diversos factores asociados a fascioliasis, entre los que destacan la crianza de animales, el consumo de vegetales crudos y el hábito de beber emolientes, entre otros; sin embargo, no se conoce con precisión los factores que predisponen a esta parasitosis en diversas zonas con casos positivos; por ejemplo, en el distrito de Condebamba, departamento de Cajamarca, se ha establecido la presencia del caracol Lymnaea neotropica, principal responsable de la infección en ganado, pero no se han reportado estudios de prevalencia de infección humana (Bargues et al., 2012). Es así, que la presente investigación tuvo como objetivo determinar la prevalencia y factores de riesgo en escolares de primaria del distrito de Condebamba, Cajamarca, Perú. 


\section{Materiales y Métodos}

\section{Localización del Estudio}

El distrito de Condebamba está ubicado en Cajamarca, región andina del norte del Perú (Figura 1). La localidad contaba con 13 988 habitantes en 2014, siendo niños entre 5 y 14 años el $21.8 \%$ de la población edad (INEI, 2015).

Más del 60\% de la población del distrito se encuentra en condiciones de pobreza, no cuentan con servicios básicos adecuados, carecen de agua potable, solo el $2 \%$ tiene red pública dentro de la vivienda, el $85 \%$ utiliza letrinas y cerca del $11 \%$ no cuenta con servicios higiénicos. En este estudio se evaluó la situación sanitaria con respecto a la fascioliasis en tres escuelas de tres localidades de Condebamba (Cauday, Ogosgón y La Merced) (Figura 1), que se encuentran geográficamente ubicadas a distintos niveles sobre el nivel del mar y presentan climatología local distinta.

\section{Criterios de Inclusión y Exclusión}

Se trabajó con niños de uno u otro sexo entre 6 y 12 años, que habían residido por lo menos seis meses en la zona a la fecha del estudio, y que se disponía del consentimiento informado y autorizado con firma del padre, madre o tutor del niño, asentimiento informado y autorizado con firma de niños desde los 9 años a más, y de haber proporcionado las muestras fecales con cuestionarios resueltos completos. Se excluyeron los niños que habían recibido tratamiento antiparasitario dentro de los 15 días previos al estudio.

\section{Tamaño Muestral}

Para determinar el tamaño muestral se utilizó el programa Epidat 3.0, tomando como referencia el $24.4 \%$ de prevalencia en niños de Cajamarca (González et al., 2011), con un nivel de confianza al $95 \%$, un margen de error

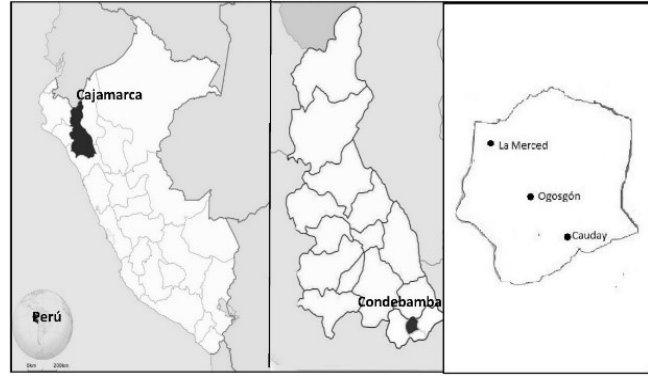

Figura 1. Área del estudio

del 5\% y una población de 2076 escolares. El total de escolares participantes fue de 253 . En base a los registros de la Dirección Regional de Educación Cajamarca, se realizó un muestreo estratificado y polietápico con asignación proporcional. En la primera etapa se determinó el número de alumnos por localidad, en la segunda etapa se seleccionaron las instituciones educativas, en la tercera etapa las aulas y en cada aula los participantes. La selección de escolares fue en forma aleatoria, usando una tabla de números aleatorios y tomando como marco muestral el listado de escolares por aula en orden alfabético.

\section{Procedimientos}

Se encuestó personalmente a los padres de familia o apoderados y a los escolares. Un cuestionario de preguntas sobre los posibles factores de riesgo para la infección por $F$. hepatica (aspectos personales, clínicos, condiciones socioeconómicas y conocimientos) fue aplicado a los primeros; un segundo cuestionario enfocado en prácticas de riesgo fue aplicado a los escolares. Ambos cuestionarios se diseñaron tomando en cuenta estudios similares (Curtale et al., 2003; Marcos et al., 2004, 2006; Fentie et al., 2013), y sus contenidos fueron validados mediante juicio de expertos y prueba piloto.

Las muestras fecales de cada escolar fueron colectadas entre agosto y diciembre de 2014 y transportadas bajo cadena de frío 
al Laboratorio de Parasitología de la Universidad Nacional de Cajamarca para el diagnóstico. Las muestras se conservaron en formol al $10 \%$, a razón de una parte de heces por tres partes de formol (Beltrán et al., 2003). Todas las muestras fueron procesadas mediante la técnica de sedimentación rápida modificada por Lumbreras y la técnica de sedimentación espontánea en tubo para el diagnóstico de $F$. hepatica (Lumbreras et al., 1962).

Para el recuento de huevos de $F$. hepatica se empleó el kit comercial «Coprokit Kato- Katz ${ }^{\circledR}$ » (Campinas Medical, Brasil). El procedimiento y lectura se realizó siguiendo las instrucciones del fabricante. Todas las muestras con diagnóstico positivo al trematodo con la técnica de sedimentación rápida fueron procesadas con este kit por triplicado para calcular la media del recuento de huevos de las tres láminas. Los resultados se expresaron en huevos por gramo de heces (hpg).

\section{Análisis Estadístico}

Los datos se ingresaron al programa estadístico SPSS v. 23. En el análisis univariado se determinó la distribución de frecuencias. La asociación entre variables cualitativas se estableció mediante la prueba de Chi cuadrado. Las asociaciones con $p<0.05$ resultantes ingresaron al análisis de regresión logística.

Para establecer los factores de riesgo asociados a la infección por $F$. hepatica se empleó la regresión logística con análisis multivariado en los casos cuando la razón de disparidad u Odds Ratio (OR) y los intervalos de confianza (IC) fueran superiores a 1 , con un $95 \%$ de confiabilidad.

Para variables cuantitativas se calcularon medidas de tendencia central y de dispersión; además, se corroboró su normalidad usando el Test de Kolmogorov-Smirnov y se aplicó $\mathrm{T}$ de Student a los datos que presenta- ron distribución normal; en caso contrario, se empleó la prueba U de Mann- Whitney. El nivel de significación estadística fue $\mathrm{p}<0.05$.

\section{Aspectos Éticos}

El protocolo de estudio fue revisado y aprobado por el Comité Institucional de Ética en Investigación del Instituto de Investigación Nutricional (La Molina, Lima). La participación fue voluntaria, se tomó el consentimiento del padre o apoderado, y el asentimiento de cada escolar participante. Los escolares con diagnóstico positivo a $F$. hepatica fueron examinados y tratados por el médico infectólogo del Hospital Regional de Cajamarca.

\section{Resultados}

Trece de 253 escolares (5.1\%) de Condebamba presentaron huevos de $F$. hepatica en las muestras fecales (Cuadro 1). Las prevalencias muestran a los escolares entre 8 y 9 años como el grupo con porcentaje ligeramente mayor de positividad, pero sin diferencias significativas. Así mismo, las niñas resultaron ser ligeramente más afectados que los niños, aunque tampoco hubo diferencia significativa (Cuadro 2). El número de hpg estuvo en el rango de 24-1380, con una media aritmética de 256 (Cuadro 3).

Entre los niños infectados por $F$. hepatica, solo en tres de ellos se pudo observar coinfección con patógenos como Giardia lamblia e Hymenolepis nana. Al haber una baja frecuencia de coinfección entre $F$. hepatica y otros parásitos, no se encontró asociación entre estos parásitos y el trematodo $(\mathrm{p}=0.53)$.

De las 15 variables ingresadas, dos de ellas mostraron asociación estadística mediante análisis univariado: crianza de vacas y defecar al aire libre, las cuales, en el análisis multivariado fueron identificadas como factores de riesgo (Cuadro 4). 
Cuadro 1. Prevalencia de infección por Fasciola hepatica en escolares de primaria del distrito de Condebamba, Cajabamba, según localidad de procedencia

\begin{tabular}{lccccc}
\hline \multirow{2}{*}{ Localidad } & \multirow{2}{*}{$\begin{array}{c}\text { Altitud } \\
(\mathrm{msnm})\end{array}$} & Total & Positivos & \multicolumn{2}{c}{ Prevalencia } \\
\cline { 5 - 6 } & 2807 & 143 & 7 & 4.9 & $1.0-8.8$ \\
Cauday & 2733 & 70 & 6 & 8.6 & $1.3-15.8$ \\
Ogosgón & 2029 & 40 & 0 & 0 & - \\
La Merced & & 253 & 13 & 5.1 & $2.2-8.1$ \\
\hline Total & & &
\end{tabular}

$\mathrm{X}^{2}=3.88 ; \mathrm{p}=0.14$

IC 95\%: Intervalo de confianza al 95\%

Cuadro 2. Prevalencia de infección por Fasciola hepatica en escolares de primaria del distrito de Condebamba, Cajabamba, según edad y género

\begin{tabular}{|c|c|c|c|}
\hline \multirow{2}{*}{ 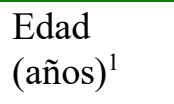 } & \multirow{2}{*}{$\frac{\text { Total }}{\mathrm{n}}$} & \multicolumn{2}{|c|}{ Prevalencia } \\
\hline & & $\%$ & IC 95\% \\
\hline $6-7$ & 49 & 4.1 & $0.5-14.0$ \\
\hline $8-9$ & 100 & 7.0 & $1.5-12.5$ \\
\hline $10-12$ & 104 & 3.8 & $1.1-9.6$ \\
\hline \multicolumn{4}{|l|}{ Género $^{2}$} \\
\hline Mujer & 132 & 6.8 & $2.1-11.5$ \\
\hline Hombre & 121 & 3.3 & $1.0-8.2$ \\
\hline
\end{tabular}

\section{Discusión}

En solo dos de las tres escuelas estudiadas en el distrito de Condebamba se encontraron casos positivos a $F$. hepatica. Las prevalencias fueron menores a $10 \%$ en ambas escuelas (Cuadro 1), por lo que las localidades de Cauday y Ogosgón, según la clasificación realizada por la OMS (Mas-Coma, 2005) pueden considerarse como áreas mesoendémicas. La ausencia de casos de $F$. hepatica en la localidad de La Merced podría explicarse por la climatología local, debido a que esa zona está ubicada a 2000-2150 msnm, con un clima cálido y poco lluvioso, que condiciona la ausencia del hospedero intermediario. Además, la población local no cría ganado vacuno, animales que cumplen un importante papel en el ciclo de trasmisión del parásito (Mas-Coma, 2005). Ambas características eco-climáticas son distintas al de las otras dos localidades en estudio.

Los hallazgos del presente estudio son similares a los descritos en otras zonas altoandinas del país. En Cusco, en un estudio en niños de 3 a 12 años se encontró $9.7 \%$ de prevalencia (Cabada et al., 2014); la prevalencia en Junín fue de $5.1 \%$ en niños de 4 a 12 años (Mantari et al., 2012), mientras que en las zonas altoandinas de Lima se encontró una prevalencia de $8.6 \%$ en una población que incluyó a niños y adultos jóvenes (Marcos et al., 2007). Asimismo, la prevalencia hallada fue similar a la reportada en otros países, como México, con 5.8\% de escolares infectados (Zumaquero-Ríos et al., 2013).

Analizando las prevalencias según grupos etarios, se observó que la prevalencia de $F$. hepatica en escolares entre 8 y 9 años fue ligeramente mayor, aunque sin diferencias significativas (Cuadro 2). Estas valores fueron similares a reportes de otras áreas endémicas andinas (Esteban et al., 1997, 
Cuadro 3. huevos de Fasciola hepatica por gramo de heces (hpg), mediante kato katz, en escolares de primaria del distrito de Condebamba, Cajamarca, según localidad de procedencia, género y grupo etario

\begin{tabular}{cccc}
\hline Variable & Rango & $\begin{array}{c}\text { Media } \\
\text { aritmética }\end{array}$ & $\begin{array}{c}\text { Media } \\
\text { geométrica }\end{array}$ \\
\hline Localidad $^{1}$ & & & \\
Cauday & $24-96$ & 34.3 & 29.3 \\
Ogosgón & $24-1380$ & 514.0 & 204.0 \\
La Merced & n.a. & n.a. & \\
Género & & & \\
Mujer & $24-1380$ & 354.7 & 105.3 \\
Hombre & $24-60$ & 33.0 & 30.2 \\
Edad (años) & & & \\
$6-7$ & $312-1212$ & 762.0 & 614.9 \\
$8-9$ & $24-1380$ & 222.9 & 48.8 \\
$10-12$ & $24-96$ & 60.0 & 48.0 \\
\hline Total & $24-1380$ & 255.7 & 71.7 \\
\hline
\end{tabular}

n.a.: no aplica (no se presentaron casos)

${ }^{1} p>0.05$

Cuadro 4. Factores asociados a la infección por Fasciola hepatica $(\mathrm{p}<0.05)$ en escolares de primaria del distrito de Condebamba, Cajabamba, mediante análisis univariado y multivariado

\begin{tabular}{|c|c|c|c|c|c|}
\hline \multirow{3}{*}{ Variable de estudio } & \multicolumn{2}{|c|}{ Análisis univariado } & \multicolumn{3}{|c|}{ Análisis multivariado } \\
\hline & $\begin{array}{l}\text { Positivo } \\
\text { (13) }\end{array}$ & $\begin{array}{c}\text { Negativo } \\
(240)\end{array}$ & OR & IC (95\%) & $\mathrm{p}$ \\
\hline & $\%$ & $\%$ & & & \\
\hline
\end{tabular}

Criar vacas en los

alrededores de vivienda

$\begin{array}{lcccccccc}\text { Sí } & 9 & 69.2 & 86 & 35.8 & 0.02 & 4.07^{*} & 1.2-13.8 & 0.024 \\ \text { No } & 4 & 30.8 & 154 & 64.2 & & - & - & \end{array}$

Defecar al aire libre

\begin{tabular}{lcccccccc} 
Sí & 7 & 53.8 & 63 & 26.2 & 0.03 & $3.32 *$ & $1.1-10.4$ & 0.040 \\
No & 6 & 46.2 & 177 & 73.8 & & - & - & \\
\hline
\end{tabular}

* Factor de riesgo 
2002; González et al., 2011; Cabada et al., 2014). Este rango de edad sería un periodo crítico e importante desde el punto de vista epidemiológico para adquirir la infección, ya que coincide con el inicio de los niños en algunas actividades laborales de ganadería y agricultura, que los predispondría a un contacto más cercano con las fuentes de infección (Marcos et al., 2002).

La mayor, aunque no significativa, prevalencia de $F$. hepatica en niñas sobre niños (Cuadro 2), coincide con otros estudios realizados en escolares (Esteban et al., 2002; González et al., 2011; Mantari et al., 2012; Zumaquero-Rios et al., 2013), donde se explica la similitud de actividades que realizan en el campo (por ejemplo, llevar a pastar al ganado) y el acceso común a fuentes de contaminación, como el consumo de vegetales crudos o ingestión de agua contaminada con metacercarias (Marcos et al., 2004; Khan et al., 2012; Mantari et al., 2012).

El número de huevos por gramo de heces es un indicador indirecto de la severidad (intensidad) de la infección por $F$. hepatica, siendo Kato-Katz el método de diagnóstico recomendado por la OMS (WHO, 2011). En este estudio, los valores hpg hallados estuvieron entre 24 y 1380 (media aritmética: 255.7; media geométrica: 71.7) (Cuadro 3), coincidiendo con el estudio realizado en Puno (Esteban et al., 1999, 2002). Si bien se encontró dos niños con recuentos mayores a 1000 hpg, el promedio no superó el límite de $400 \mathrm{hpg}$, que suele ser usado para identificar infecciones de alta intensidad (WHO, 2007). No se encontraron diferencias significativas en las intensidades de infección en relación con la procedencia, género y grupo etario, lo que puede deberse al bajo número de casos positivos, coincidiendo con investigaciones realizadas en Perú y en Egipto (Esteban et al., 2002, 2003).
En el análisis univariado y multivariado (Cuadro 4) se encontró asociación significativa entre la crianza de vacas en los alrededores de la vivienda y la infección por $F$. hepatica, coincidiendo con una investigación realizada en el norte de Etiopía, en el cual la presencia de ovejas y bovinos estuvo significativamente asociada con el incremento del riesgo de Fasciola sp (Fentie et al., 2013). Así mismo, otros estudios han demostrado la asociación entre el trematodo y la crianza de bovinos cerca de la vivienda (Curtale et al., 2003; Marcos et al., 2006), lo que sustenta la importancia de controlar la infección en los rumiantes domésticos, principalmente en las áreas endémicas de fascioliasis humana (Curtale et al., 2003).

La práctica de defecar al aire libre como factor de riesgo para los niños (Cuadro 4), confirma la posibilidad de un ciclo humano a humano en la transmisión del parásito, sobre todo si la eliminación de huevos de $F$. hepatica por las personas resulta ser lo suficientemente alta, y estos huevos han demostrado ser viables (Mas-Coma et al., 1999). Así mismo, es fácil asumir que los niños no tienen buenos hábitos higiénicos si suelen defecar en el campo y, por tanto, hay mayor probabilidad de contacto con el ambiente contaminado y la fuente de infección (Marcos et al., 2006).

El diagnóstico de $F$. hepatica mediante la técnica de sedimentación rápida descrita por Lumbreras constituye la herramienta convencional de elección, ya que ofrece mejores características para el diagnóstico de $F$. hepatica: mayor sensibilidad, reproducibilidad, adaptabilidad y sencillez, utiliza materiales de bajo costo y fácil acceso y brinda resultados confiables que facilitan la orientación de un oportuno esquema terapéutico por parte del médico tratante (Maco et al., 2002; Uribe et al., 2012). Sin embargo, es impor- 
tante resaltar que con el uso de esta técnica podría no haberse detectado algunos casos positivos debido a su limitación para el diagnóstico en fase aguda y la eliminación intermitente de huevos por el trematodo adulto.

\section{Conclusiones}

Se resalta la moderada prevalencia de infección por $F$. hepatica en escolares de las localidades de Cauday y Ogosgón del distrito de Condebamba, Cajamarca, donde los factores de riesgo asociados más importantes a la infección serían la crianza de ganado bovino y las prácticas de defecación de los niños al aire libre.

\section{Literatura Citada}

1. Bargues MD, Artigas $P$, Khoubbane M, Ortiz P, Naquira C, Mas-Coma S. 2012. Molecular characterisation of Galba truncatula, Lymnaea neotropica and L. schirazensis from Cajamarca, Peru and their potential role in transmission of human and animal fascioliasis. Parasite Vector 5: 174-190. doi: 10.1186/1756-3305-5-174

2. Beltrán M, Tello R, Náquira C. 2003. Manual de procedimientos de laboratorio para el diagnóstico de los parásitos intestinales del hombre. Serie de Normas Técnicas N. ${ }^{\circ} 37$. Lima: Instituto Nacional de Salud. [Internet]. Disponible en: http://www.bvs.ins.gob.pe/ insprint/salud_publica/nor_tec/37.pdf

3. Cabada MM, Goodrich MR, Graham B, Villanueva-Meyer PG, Lopez M, Arque E, White AC. 2014. Fascioliasis and eosinophilia in the highlands of Cuzco, Peru and their association with water and socioeconomic factors. Am J Trop Med Hyg 91: 989-993. doi: 10.4269/ ajtmh.14-0169
4. Curtale F, Mas-Coma S, Hassanein YA, Barduagni P, Pezzotti P, Savioli L. 2003. Clinical signs and household characteristics associated with human fascioliasis among rural population in Egypt: a case-control study. Parassitologia 45: 5-11.

5. Cwiklinski K, O'neill SM, Donnelly S, Dalton JP. 2016. A prospective view of animal and human fasciolosis. Parasite Immunol 38: 558-568. doi: 10.1111/ pim. 12343

6. Espinoza J, Terashima A, HerreraVelit P, Marcos LA. 2010. Fasciolosis humana y animal en el Perú: impacto en la economía de las zonas endémicas. Rev Perú Med Exp Salud Pública 27: 604-612.

7. Esteban JG, Flores A, Aguirre C, Strauss W, Angles R, Mas-Coma S. 1997. Presence of very high prevalence and intensity of infection with Fasciola hepatica among Aymara children from the Northern Bolivian Altiplano. Acta Trop 66: 1-14. doi: 10.1016/S0001706X(97)00669-4

8. Esteban JG, Flores A, Angles R, MasComa S. 1999. High endemicity of human fascioliasis between Lake Titicaca and La Paz valley, Bolivia. T Roy Soc Trop Med H 93: 151-156. doi: 10.1016/S0035-9203(99)90289-4

9. Esteban JG, Gonzalez C, Bargues MD, Angles R, Sanchez C, Naquira C, Mas-Coma S. 2002. High fascioliasis infection in children linked to a manmade irrigation zone in Peru. Trop Med Int Health 7: 339-348. doi: 10.1046/ j.1365-3156.2002.00870.x

10. Esteban JG, González LC, Curtale F, Muñoz-Antoli C, Valero MA, Bargues $M D$, et al. 2003. Hyperendemic fascioliasis associated with schistosomiasis in villages in the Nile Delta of Egypt. Am J Trop Med Hyg 69: 429-437.

11. Fentie T, Erqou S, Gedefaw M, Desta A. 2013. Epidemiology of human fascioliasis and intestinal parasitosis among schoolchildren in Lake Tana 
Basin, northwest Ethiopia. T Roy Soc Trop Med H 107: 480-486. doi: 10.1093/ trstmh/trt056

12. González LC, Esteban JG, Bargues $M D$, Valero MA, Ortiz P, Náquira C, Mas-Coma S. 2011. Hyperendemic human fascioliasis in Andean valleys: an altitudinal transect analysis in children of Cajamarca province, Peru. Acta Trop 120: 119-129. doi: 10.1016/j.actatropica.2011.07.002

13. [INEI] Instituto Nacional de Estadística e Informática. 2015. Compendio estadístico Perú 2014. Lima, Perú: INEI. [Internet]. Disponible en: https:// w w w. i n e i.gob.pe/media/ MenuRecursivo/publicaciones digitales/ Est/Lib1173/compendio2014.html

14. Khan I, Khan AM, Ayaz, K, Anees M, Khan SA. 2012. Molecular detection of Fasciola hepatica in water sources of district Nowshehra Khyber Pakhtunkhwa Pakistan. Int J Adv Res Technol 1: 106-117.

15. Lumbreras $H$, Cantella $R$, Burga $R$. 1962. Acerca de un procedimiento de sedimentación rápida para investigar huevos de Fasciola hepatica en las heces, su evaluación y uso en el campo. Rev Médica Perú 31: 167-174.

16. Maco V, Marcos L, Terashima A, Samalvides F, Miranda E, Espinoza J, Gotuzzo E. 2002. Fas2-ELISA y la técnica de sedimentación rápida modificada por Lumbreras en el diagnóstico de la infección por Fasciola hepatica. Rev Med Hered 13: 49-57.

17. Mantari C, Chávez A, Suárez F, Arana C, Pinedo R, Ccenta R. 2012. Fasciolasis en niños de tres distritos del departamento de Junín, Perú. Rev Inv Vet Perú 23: 454-461. doi: 10.15381/ rivep.v23i4.952

18. Marcos LA, Maco V, Terashima Al, Samalvides F, Gotuzzo E. 2002. Características clínicas de la infección crónica por Fasciola hepatica en niños. Rev Gastroenterol Perú 22: 228-233.
19. Marcos LA, Maco V, Terashima A, Samalvides $F$, Miranda E, Tantalean M, Espinoza J, et al. 2004. Hiperendemicidad de fasciolosis humana en el valle del Mantaro, Perú: factores de riesgo de la infección por Fasciola hepatica. Rev Gastroenterol Perú 24: 158-164.

20. Marcos L, Maco V, Samalvides F, Terashima A, Espinoza JR, Gotuzzo E. 2006. Risk factors for Fasciola hepatica infection in children: a casecontrol study. T Roy Soc Trop Med H 100: 158-166. doi: 10.1016/j.trstmh.2005.05 .016

21. Marcos L, Romani LF, Florencio L, Terashima A, Canales M, Nestares J, Huayanay L, et al. 2007. Zonas hiperendémicas y mesoendémicas de la infección por Fasciola hepatica aledañas a la ciudad de Lima: una enfermedad emergente. Rev Gastroenterol Perú 27:31-36.

22. Marcos LA, Terashima A. 2007. Update on human fascioliasis in Peru: diagnosis, treatment and clinical classification proposal. Neotrop Helminthol 1: 85-104.

23. Mas-Coma S. 2005. Epidemiology of fascioliasis in human endemic areas. J Helminthol 79: 207-216. doi: 10.1079/ JOH2005296

24. Mas-Coma MS, Esteban JG, Bargues MD. 1999. Epidemiology of human fascioliasis: a review and proposed new classification. B World Health Organ 77: 340-346.

25. Uribe N, Sierra RF, Espinosa CTE. 2012. Comparación de las técnicas Kato-Katz, TSET y TSR en el diagnóstico de infección por Fasciola hepatica en humanos. Rev Univ Ind Santander Salud 44: 7-12.

26. [WHO] World Health Organization. 2007. Report of the WHO. Informal Meeting on use of triclabendazole in fascioliasis control. Geneva, Switzerland: WHO. [Internet]. Available in: http:// 
www.who.int/neglected_diseases/ preventive_chemotherapy/ WHO_CDS_NTD_PCT_2007.1.pdf

27. [WHO] World Health Organization. 2011. Report of the WHO expert consultation on foodborne trematode infections and taeniasis/cysticercosis. Vientiane, Lao People's Democratic Republic: World Health Organization. [Internet]. Available in: http:// www.who.int/neglected_diseases/ preventive_chemotherapy/ WHO_HTM_NT̄D_PCT_2011.3.pdf

28. Zumaquero-Ríos JL, Sarracent-Pérez J, Rojas-García R, Rojas-Rivero L, Martínez-Tovilla Y, Valero MA, MasComa S. 2013. Fascioliasis and intestinal parasitoses affecting schoolchildren in Atlixco, Puebla State, Mexico: epidemiology and treatment with nitazoxanide. Plos Neglect Trop D 7: e2553. doi: 10.1371/journal.pntd.0002553 